Review

\title{
Dysphagia in the Older Age Setting
}

Asaad G Ishak ${ }^{1,}{ }^{*}$, Sundar V Cherukuri ${ }^{1}$, Giancarlo Diaz ${ }^{2}$, Richard McCallum ${ }^{3}$

1. Department of Internal Medicine, Texas Tech University Health Sciences Center, 4800 Alberta Avenue, El Paso, TX 79905, USA; E-Mails: asaad.ishak@ttuhsc.edu; sundar.cherukuri@ttuhsc.edu

2. Paul L. Foster School of Medicine (PLFSOM), Texas Tech University Health Sciences Center El Paso, 5001 El Paso Dr, El Paso, TX 79905, USA; E-Mail: giancarlo.diaz@ttuhsc.edu

3. Division of Gastroenterology, Texas Tech University Health Sciences Center, 4800 Alberta Avenue, El Paso, TX 79905, USA; E-Mail: Richard.McCallum@ttuhsc.edu

* Correspondence: Asaad G Ishak; E-Mail: asaad.ishak@ttuhsc.edu

Academic Editor: David G Smithard

Correction: Dysphagia in the Elderly

\section{OBM Geriatrics}

2021, volume 5, issue 3

doi:10.21926/obm.geriatr.2103174
Received: January 25, 2021

Accepted: June 20, 2021

Published: July 02, 2021

\begin{abstract}
Dysphagia, or difficulty swallowing, is a common condition, especially among the elderly population and can become debilitating. As we age, there are subtle changes in esophageal motility, but by no means is dysphagia ever considered to be a normal result of aging. Dysphagia usually presents with a variety of accompanying symptoms ranging from regurgitation during meals, mild discomfort, to severe chest pain. It can be challenging to localize the dysphagia from the history. The underlying etiology may become evident during subsequent investigations. It is essential to be able diagnose dysphagia as early detection is associated with decreased morbidity and in some cases mortality. In addition, early detection through clinical suspicion, confirmatory imaging or endoscopic evaluation is important as it can lead to early intervention with proper management depending on the underlying etiology, resulting in economic benefit and reduced medical costs. There are various causes of dysphagia, with the majority being benign, but a physician should always be aware of red flags present which may signal more serious causes, such as an underlying malignancy. Our goal in
\end{abstract}

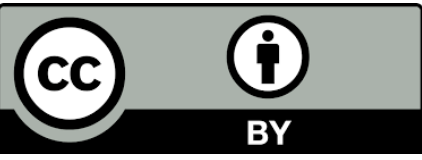

(C) 2021 by the author. This is an open access article distributed under the conditions of the Creative Commons by Attribution License, which permits unrestricted use, distribution, and reproduction in any medium or format, provided the original work is correctly cited. 
this review article is to focus on detecting the clinical signs of dysphagia and review appropriate further evaluation and update on management of this potentially serious condition in order for elderly patients to achieve an optimal quality of life.

\section{Keywords}

Esophageal dysphagia; oropharyngeal dysphagia; aging; elderly; swallow mechanism

\section{Introduction}

It is vital for individuals to receive nutrients through eating and drinking in order to meet the everyday metabolic demands required by the human body. Dysphagia, which by definition is any mechanism that disrupts the normal swallowing process, poses a challenge to meeting these needs [1]. Anatomically, dysphagia can be divided into two broad categories: oropharyngeal dysphagia and esophageal dysphagia. Oropharyngeal dysphagia, also termed "transfer dysphagia", involves difficulty efficiently forming a food bolus and moving it into the esophagus relying on striated muscle functions. Esophageal dysphagia, on the other hand, is related to inefficient or noncoordinated peristalsis of food by the smooth muscle of the esophagus delaying passage of the bolus into the stomach [2].

\section{Epidemiology (Incidence and Prevalence)}

Although it is difficult to assess the true prevalence of dysphagia given that many patients experience very mild symptoms for which many do not seek care, estimates show that in the United States alone, dysphagia affects roughly 300,000 - 600,000 individuals each year, and approximately $15 \%$ of the elderly population [1]. Another study suggests that the prevalence of dysphagia is even higher than previously calculated, stating that about $40-60 \%$ of US nursing home residents have some form of clinical evidence of dysphagia [3]. A study performed in 2013 demonstrated that the estimated incidence of dysphagia present in nursing home patients was between $20 \%$ and $40 \%$, although these numbers are difficult to stand by with certainty due to the lack of consensus for a standard assessment for this condition [4].

\section{Physiology of Normal Swallowing}

The physiology of swallowing is a complex process involving more than 30 nerves and numerous muscles, and essentially can be broken down into 3 phases: The oral, pharyngeal, and the esophageal phase. Only the oral phase is under entirely voluntary control, while the pharyngeal and esophageal phases are largely involuntary processes [5]. The oral phase involves preparing food for propulsion into the pharynx, utilizing cranial nerves V (trigeminal), VII (facial), and XII (hypoglossal) [6]. Preparing food involves mastication and mixing saliva with the food, converting it into a bolus in the process, which makes it easier to propel food into the stomach to undergo trituration [7]. The tongue is a very important component of the oral phase as its contour and movement is what seals and gives the food bolus its shape [5]. Next is the pharyngeal phase, which seals the pharynx by elevation of the larynx, specifically the crycothyroid muscle, preventing aspiration and facilitating 
projection of the food bolus into the esophagus. This involves the reflexive use of cranial nerves $\mathrm{V}$ (trigeminal), X (vagus), XI (accessory), and XII (hypoglossal) [6]. Through a delicate and coordinated process driven by striated muscles, the food bolus progresses from the pharynx across the upper esophageal sphincter (UES) and into the cervical esophagus [8]. The final phase of swallowing, known as the esophageal phase, involves the smooth muscles and the process of coordination called peristalsis whereby food descends through the esophagus while there is relaxation of the lower esophageal sphincter (LES) so that food may enter the stomach [6].

\section{Effects of Aging on Normal Swallowing}

As with all other organs and functions of the human body, aging also has physiological changes affecting swallowing and while significant clinical esophageal dysfunction is not a direct result of aging, mild manometric changes have been described in the smooth muscle of the esophagus. Alterations in the elderly include a decrease in the amplitude of esophageal smooth muscle contractions, the number of peristaltic waves following a swallow, increase in disorganized or nonperistaltic contractions in the body of the esophagus and weakening of the esophageal smooth muscle [9].

Oropharyngeal dysphagia results from a wide variety of subtle changes in relaxation of the UES or strength of the pharyngeal function. UES function, which is mainly composed of the cricopharyngeus muscle, can be observed through videofluoroscopic studies, and small changes have been observed [9].

In the smooth muscle, the secondary esophageal peristalsis is less frequent with advanced age and, in some cases, absent after esophageal distension. It is believed that the decreased amplitude of esophageal contractions is due to a reduced number of cells in the enteric nervous system attributed to the underlying diabetes or vascular changes that occur with age [9].

Other factors to consider are the reduction in both muscle mass and connective tissue elasticity leading to a loss of range of motion and strength which hinders the ability to efficiently propel food boluses through the upper aerodigestive tract [1].

In addition, elderly individuals have decreased mastication function and production of saliva for lubrication, leading to sub-optimal propulsion of the food bolus [2].

In a barium swallow study composed of 56 individuals with a mean age of 83 years who were asymptomatic, $84 \%$ of patients demonstrated videofluoroscopic changes in pharyngeal swallowing function compared to their younger counterparts [10]. Oral transit time was found to decrease with age and altered function in the pharyngoesophageal segment was six times more common in the elderly. Despite this, it is challenging to determine whether impaired swallowing in the elderly is due to normal aging, subclinical disease, or a disease early in its pathological state [10].

\section{Clinical Signs and Symptoms}

There are several symptoms, or characteristics, that help differentiate oropharyngeal from esophageal dysphagia. Oropharyngeal dysphagia tends to present with: nasopharyngeal regurgitation, aspiration, coughing while eating, drooling, dysphonia, and is more common among patients with neurological diseases. Physical findings indicative of oropharyngeal dysphagia may include: an oropharyngeal mass suggesting a Zenker's diverticulum (particularly when the mass is protruding to the left side of the neck), ulceration of the skin secondary to damage from radiation 
for head and neck tumors, neck mass that can raise concerns for malignancy, or cervical adenopathy [9].

Esophageal dysphagia typically presents with symptoms to either solids which may progress to liquids as well such as: acid reflux or heartburn, a peptic stricture, regurgitation, chest pain and weight loss, or onset initially for dysphagia to both solids and liquids for example in a motility disorder, namely achalasia, scleroderma, or diffuse esophageal spasms [9].

While dysphagia can sometimes be easily diagnosed, (e.g., when a patient immediately chokes upon swallowing), symptoms are more frequently insidious and under-reported in the elderly population. Therefore, this can make diagnosing dysphagia in this age group challenging, especially in individuals with speech or cognitive impairments. As with many other conditions, the first step in assessing dysphagia begins with simple bedside observation. There are several signs that point towards a diagnosis of dysphagia. These include: prolonged duration of mealtime or prolonged chewing, food remnants in the oral cavity after eating, nasal reflux of food, slobbering during a meal, sensation of blockage during swallowing, and changes in voice quality. Other symptoms also include interrupted respirations or coughing during meals (including aspiration episodes), reduction or refusal of food intake, changes in preferred meal textures, recurrent pulmonary infections, and unexplained episodes of fever and/or unintentional weight loss [11].

Localization is paramount when attempting to distinguish oropharyngeal vs esophageal dysphagia, but this can be challenging due to the phenomena of referred pain related to the $4^{\text {th }}$ cervical nerve distribution that is involved in both the diaphragmatic and the cricopharyngeal innervation. Most patients with esophageal dysphagia feel their discomfort in the suprasternal notch or lower neck, yet the obstruction tends to be much lower in the chest. Therefore, when patients present with dysphagia referred to this location, it should be considered non-informative. On the other hand, dysphagia that causes discomfort below the suprasternal notch provides a more reliable localization of the obstruction or source [9].

Dysphagia should be considered an alarm symptom given the possibility of an underlying malignancy. There are several symptoms which should prompt the physician to order immediate diagnostic studies including: weight loss which is a sign of advanced disease with over 10 percent of total body mass [12], bleeding, and odynophagia (a term used to describe painful swallowing to solids and liquids) [13].

The syndrome termed Plummer- Vinson syndrome, or Kelly-Patterson, usually presents in females with dysphagia, an upper esophageal web or stricture, and iron deficiency anemia are characteristic features of the syndrome. These are often attributed to impaired nutrition from the dysphagia, plus an autoimmune phenomena such as Celiac disease (Sprue) or antiparietal or antimuscular antibody associated with a positive ANA, impairing Iron absorption [14].

Rarely does occult esophageal bleeding cause iron deficiency anemia. There are several red flag symptoms which should raise concern for malignancy. These symptoms include anorexia, dysgeusia, and progression from solid to liquid dysphagia. Dysphagia associated with long-standing acid reflux symptoms would lead to considering a peptic stricture, Barrett's esophagus or malignancy [9]. 


\section{Clinical Overview}

As previously mentioned, dysphagia can be broken down clinically into two main categories: oropharyngeal dysphagia and esophageal dysphagia. Each of these can include both functional and structural disorders as underlying causes of dysphagia.

The first step in evaluating patients with dysphagia is to determine whether it is oropharyngeal dysphagia (transfer) or esophageal (Tubular) dysphagia. As with any medical condition, it is essential to obtain a thorough history and physical exam. Generally, difficulty initiating swallowing points towards oropharyngeal dysphagia, while having a sensation of food stopping or sticking after swallowing is more characteristic of esophageal dysphagia. It is also important to determine which foods are difficult to swallow (solids vs. liquids vs. both), if the dysphagia is intermittent, constant, or progressively worse, where the location of the sensation of food being trapped, and if there are any other associated symptoms such as chest pain, heartburn, regurgitation, hoarseness, and weight loss. Oropharyngeal dysphagia predominately occurs with liquids, while esophageal dysphagia more commonly occurs with solids. If dysphagia is caused by both solids and liquids from the start with regurgitation of food and saliva then achalasia might be considered as an etiology (Birgisson S, Richter). Pre-existing medical conditions such as scleroderma and use of medications are also important to ask for when gathering a history from the patient. If the symptoms arose suddenly, there is less likely to be an underlying malignant etiology, while symptoms that are progressive in nature or progress from solids to liquids high a higher likelihood of being due to a stricturing process including an underlying malignancy [8].

When suspecting esophageal dysphagia, it is important to thoroughly inspect the skin of the patient, as dysphagia can be association with CREST syndrome. Findings of CREST syndrome include: telangiectasia, Raynaud's phenomenon (GE reflux and Sjogren's syndrome), calcinosis and scleroderma impairing smooth muscle motility and function in the esophagus. Patients with suspected esophageal dysphagia accompanied by allergy symptoms such as allergic conjunctivitis, eczema, asthma, should raise the suspicion for eosinophilic esophagitis. In immunocompromised patients such as individuals who are HIV positive or those on immunosuppressive medications, it is always essential to inquire about accompanying odynophagia and rule out infectious etiologies, including herpes, CMV, and candida [9].

As previously stated, adverse effects of medications (please refer to Table 1) are an underappreciated cause of dysphagia, and therefore thorough medication reconciliation and review is warranted, along with a focused investigation of substernal pain, odynophagia, and dysphagia [9]. Since neurological disorders are a common cause of dysphagia in the elderly, every patient with dysphagia should undergo a neurological examination with the option to proceed with rapid referral to a speech and language therapist for the timely assessment and management [7].

Table 1 Medications that may cause dysphagia/odynophagia.

\begin{tabular}{ll}
\hline Psychiatric/neurologic drugs & Benzodiazepines, tricyclic antidepressants, antihistamines \\
Narcotics & Opioids (morphine, fentanyl, codeine, hydromorphone, etc.) \\
Supplements & Potassium chloride, ascorbic acid \\
Antibiotics & Doxycycline \\
Bisphosphonates & Alendronate
\end{tabular}




$\begin{array}{ll}\text { NSAIDs } & \text { Aspirin } \\ \text { Antiarrhythmic agents } & \text { Quinidine } \\ \text { Anti-epileptics } & \text { Phenytoin }\end{array}$

Rehabilitation involves a multifaceted approach with the usage of numerous techniques and treatment combinations to help improve swallowing function. These include, but are not limited to, neuromuscular electrical stimulation, laryngeal elevation exercises, effortful swallows, oromotor exercises, and oral bolus trials [15]. Routine therapy sessions are recommended with multiple techniques used per session.

\section{Diagnostic Testing}

According to the American College of Radiology, the optimal imaging study to diagnose dysphagia is dependent on the nature, location, and clinical location of the patient's dysphagia. In most cases, the gold standard for diagnosing dysphagia suspected of oropharyngeal etiology remains to be the videofluoroscopic study [16]. A modified barium swallow study is a videofluoroscopic procedure which evaluates a patient's oropharyngeal swallow. During this procedure, a speech therapist is present with the radiologist and a mixture of apple sauce or oatmeal is mixed with thick barium. A modified barium swallow study (BSS) specifically focuses on the oral cavity, pharynx, and cervical esophagus to assess abnormalities in both the oral and pharyngeal phases of swallowing. This allows for assessment of bolus manipulation, tongue motion, hyoid, laryngeal, and pharyngeal elevation, soft-palate elevation, pharyngeal constrictor motion, epiglottic tilt, laryngeal penetration, cricopharyngeal muscle function including relaxation, Zenker's diverticulum, and the potential for aspiration [17].

A BSS is the appropriate first step when esophageal dysphagia is suspected because it is sensitive in detecting both extrinsic obstructions, such as a mediastinal mass, lymph nodes and blood vessels, and intrinsic obstructive lesions, such as peptic strictures, esophageal ring, esophageal dilation, achalasia, esophageal web, and cancerous lesions. In addition, barium sulfate tablets have also been used to diagnose esophageal narrowing not fully appreciated by just barium study. The tablet size of $12.5 \mathrm{~mm}$ has been appropriate; given the association between the degree of dysphagic sensation and the reduction of esophageal lumen diameter below $13 \mathrm{~mm}$. Tablets are "held up" in the esophagus at the site of the lesion causing dysphagia. If a lesion is evident on a BSS, then an upper gastrointestinal flexible endoscopy with a biopsy and brush cytology is warranted [18].

If there is no evidence of anatomical obstructions on imaging, then endoscopic evaluations are considered necessary to make sure lesions are not overlooked. The fiberoptic endoscopic evaluation of swallowing (FEES) protocol can also help evaluate the upper pharyngoesophageal junction [19]. The protocol serves a similar purpose to a BSS, in identifying the process of swallowing and to test the ability and response to alterations in the process. Utilizing a fiberoptic scope, the anatomy of the upper aerodigestive tract is evaluated for any deficits or excess secretions that may contribute to dysphagia. Structural motions are also assessed in non-swallow phonatory tasks, such as laryngeal elevation and closure. These motions are re-evaluated in swallow tasks to evaluate for dysfunction. Sensory testing is performed by asking patients if they feel or respond with coughing to certain objects in the throat, such as foods, liquids, or the endoscope itself. A small jet of air can also be pushed to invoke a laryngeal adductor reflex, which modifies the protocol into a FEES 
sensory test (FEEST). The last portion of the FEES examination involves a direct assessment of food and liquid swallowing. It helps to assess for aspiration, bolus clearance time, and bolus characteristics, like a BSS [19].

Transnasal esophagoscopy is a rising investigative method utilizing the nasal passage in the outpatient setting to examine the upper aerodigestive tract. It is usually well-tolerated without procedural sedation and has been proposed as an initial investigative step for dysphagia [20]. An alternative approach is to undergo a complete upper endoscopic evaluation with biopsies to also assess for eosinophilic esophagitis, gastroesophageal reflux, and Barrett's esophagus.

If these studies are not conclusive then a high-resolution esophageal manometry may be performed to assess esophageal motility. Manometry measures the pressures and patterns of peristalsis of the esophagus, or the lack of it, and can assess UES and LES responses to swallowing. Esophageal $\mathrm{pH}$ monitoring and esophageal impedance studies are other techniques that can be used to determine if acid reflux is present and potentially contributing to the dysphagia by inducing "esophageal mucosa sensitivity" [8]. These methods allow for evaluation of reflux at the level of the pharynx and can help determine correlations between esophageal motility, reflux, and dysfunction.

Scintigraphy is another method that has been shown to be beneficial in evaluating laryngopharyngeal and esophageal reflux. Studies have shown it to be useful when manometry is not tolerated, unavailable, or negative but with high suspicion for disease [21]. It also has served to help measure the outcomes of therapeutic interventions.

Additional studies can be performed in the electrophysiological characteristics of involved musculature, which cannot be analyzed solely through imaging or endoscopic studies. The activity of the suprahyoid muscle complex and the cricopharyngeal muscle, which are heavily involved in the function of the UES, are analyzed using electromyographic (EMG) recordings. In normal swallowing function, there is an inhibitory pause that allows for UES relaxation. Studies are currently investigating interventional options to restore this pause in patients with dysphagia utilizing methods such as botulism toxin injection $[22,23]$.

\section{Etiologies of Oropharyngeal Dysphagia, Prognosis and Management: Table 2A}

Table 2A Etiologies of Oropharyngeal dysphagia.

\begin{tabular}{ll}
\hline \multirow{2}{*}{ Neuromuscular Causes } & $\begin{array}{l}\text { Stroke, Dementia, Diabetes, Alzheimer's disease, Myasthenia gravis, } \\
\text { Parkinson's disease, Amyotrophic lateral sclerosis (ALS), Multiple } \\
\text { sclerosis, Huntington's disease, Muscular dystrophy }\end{array}$ \\
\hline $\begin{array}{l}\text { Structural } \\
\text { Disorders }\end{array}$ & (Mechanical) \\
$\begin{array}{l}\text { Zenker diverticulum, cricopharyngeal bar/achalasia, oropharyngeal } \\
\text { and laryngeal tumors, head and neck tumors, radiotherapy, extrinsic } \\
\text { compression (cervical osteophytes and skeletal abnormalities). }\end{array}$ \\
\hline
\end{tabular}

\subsection{Stroke}

$16.5 \%$ to $50 \%$ is the incidence rate of dysphagia in the mixed stroke patients [24]. Given the aging of our population, neurological diseases are becoming more prevalent in the elderly and thus are contributing to many new cases of dysphagia. According to The Internet Stroke Center which uses data collected by the U.S. Centers for Disease Control and Prevention, stroke is the leading cause of serious, long-term disability in the United States with dysphagia being one of these disabilities. 
Nearly $75 \%$ of strokes occur in people over the 65 , as the risk of stroke doubles each decade after the age of 55. Dysphagia as a result of stroke has been associated with an increased risk for pulmonary complications and in severe cases, even death. "Cerebral, cerebellar, or brain stem strokes can impair swallowing physiology" [25]. Cerebral lesions have also been known to disrupt the voluntary control of mastication including the transport of the food bolus during the oral phase of swallowing. Cortical lesions involving the precentral gyrus produce contralateral impairment in facial, lip, and tongue motor control, as well as contralateral impairment of pharyngeal peristalsis. While brain stem strokes are less common than cerebral and cortical cerebrovascular accidents, they cause the greatest impairment in swallowing, as they affect sensation of the mouth, tongue, cheek, timing of the initiation of pharyngeal swallow, laryngeal elevation, glottic closure, and cricopharyngeal relaxation. When dealing with strokes as an etiology of dysphagia, it is essential to understand that depending on the stroke type and patient age, multiple aspects of swallowing may be affected [25]. In stroke patients experiencing symptoms of dysphagia, it is essential to assess the need for percutaneous endoscopic gastrostomy (PEG) tube placement, which can provide temporary maintenance of nutrition and medication administration until there is some return of function, while reducing the risk of aspiration [26]. Other rehabilitation techniques, as mentioned above, can help with progressive return of function through a coordinated effort.

\subsection{Parkinson's Disease}

Parkinson's disease is another neurological disease that may cause dysphagia. This disease leads to dysfunction of the dopaminergic neural network which affects the supramedullary swallowing system and leads to dysphagia. One study suggests that progression of disease in the CNS as a result of PD possibly affects the pharyngeal muscles responsible for swallowing [27]. Another study which examined the fiber histochemistry of the pharyngeal constrictor and cricopharyngeal sphincter muscles in postmortem patients, it was demonstrated that Parkinson patients had many more atrophic fibers, fiber type grouping, and fast-to-slow myosin heavy chain transformation compared to their age-matched subjects without PD. This indicates that the pharyngeal muscles of PDs patients undergo neural degeneration and regeneration over the course of their disease [28]. Patients can require (PEG) tubes as well to prevent aspiration [26], In addition, the role of antiparkinson's disease therapy (Dopamine) to reduce the risks of dementia and help overall functional capacity.

\subsection{Xerostomia}

Xerostomia, or the subjective sensation of dry mouth, results from the decreased saliva production, which may be due to exocrine gland atrophy, and affects $29-57 \%$ of older individuals. Radiation therapy to head and neck cancer often causes salivary gland dysfunction and Xerostomia [29]. Xerostomia can also present as a symptom of an underlying condition such as Sjogren's syndrome [30]. One of the manifestations of Xerostomia is in fact dysphagia. Age in itself is not believed to be the direct cause of decreased salivary secretion, but the loss of teeth does contribute indirectly to decreased stimulated salivary flow. The most common cause of Xerostomia is adverse effects of medications, such as: tricyclic antidepressants, serotonin reuptake inhibitors, antipsychotics, antihistamines, and anticholinergics [9]. Pilocarpine tablets or drops (a muscarinic cholinergic agonist) can be useful in stimulating salivary production [31]. 


\subsection{Structural Causes}

Zenker's diverticulum (figure $1 \mathrm{~A}$ ) is a "pulsion diverticulum" and a rare condition that most commonly occurs in the elderly in Killian's triangle (the area with the highest susceptibility to create a diverticulum due to its low resistance), typically on the left side of the neck [32] (Figure 1B). Treatment for this condition is indicated for all symptomatic patients. Traditionally, Surgical approach to resect the diverticulum and perform a cricopharyngeal myotomy is successful in treating (ZD). Endoscopic treatment may be more beneficial for high-risk elderly patients who can benefit from a brief procedure without general anesthesia and without the need for hyperextension of the neck [33].
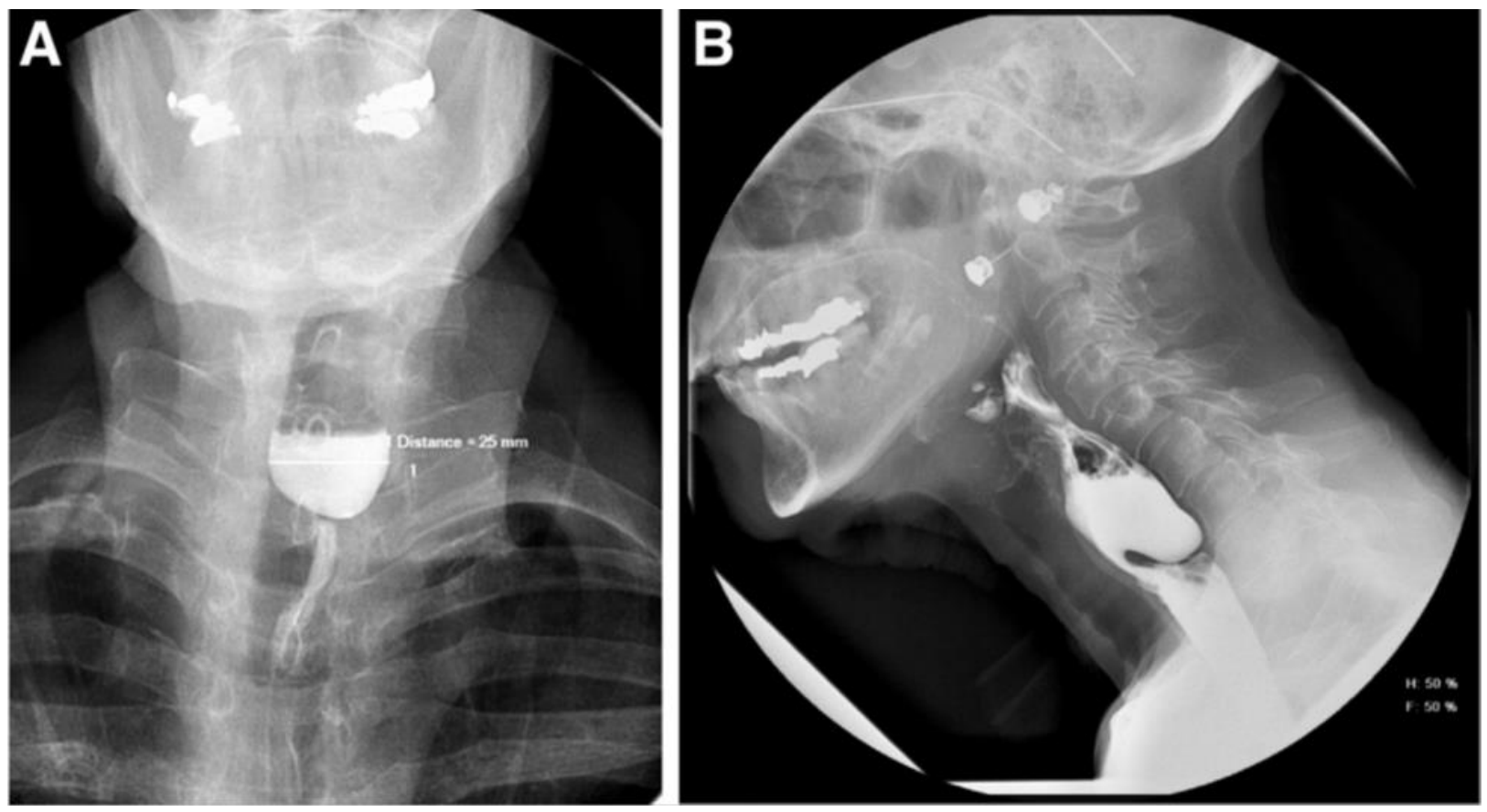

Figure 1 Radiographic imaging of Zenker's diverticulum. A) Frontal view demonstrating location modality proximal to the upper esophageal apparatus. B) Lateral view emphasizing the predominant left sided location.

\subsection{Cricophayngeal Bar}

The UES is a complex structure that is composed of the cricopharyngeus, the inferior pharyngeal constrictor, and the proximal cervical esophagus, but the main component is the cricopharyngeal (CP) muscle. A cricopharyngeal bar or "transfer dysphagia", which is difficult to appreciate on endoscopic examination, is found in $5-19 \%$ of patients who undergo pharyngeal radiography. CP bars are commonly detected in asymptomatic patients, and therefore are a diagnosis of exclusion. Histopathologically, these bars have demonstrated cricopharyngeal muscle fiber degeneration and interstitial fibrosis [34]. Treatment for CP bars is endoscopic dilation and sometimes surgery (myotomy) to overcome the fibrosis of the cricopharyngeal muscle [34]. 


\section{Major Etiologies of Esophageal Dysphagia and Management Options: Table 2B}

Table 2 B Etiologies of Esophageal dysphagia.

\begin{tabular}{ll}
\hline & $\begin{array}{l}\text { Esophageal webs, Esophageal rings (including Schatzki rings type A and B), } \\
\text { Peptic and radiation strictures, Vascular compression (dysphagia aortica, } \\
\text { Structural Causes }\end{array}$ \\
& $\begin{array}{l}\text { dysphagia lusoria) and extrinsic compression from mediastenal lesions, } \\
\text { large hiatal hernia, esophageal adenocarcinoma from long-standing } \\
\text { Barrett's esophagus, or esophageal squamous cell carcinoma }\end{array}$ \\
\hline \multirow{5}{*}{ Functional Causes } & $\begin{array}{l}\text { Erosive esophagitis, Eosinophilic esophagitis, candida and Herpes } \\
\text { esophagitis, Achalasia, Diffuse esophageal spasm, GE junction outlet } \\
\text { obstruction, hypertensive esophageal motility disorder, ineffective } \\
\text { esophageal motility, Presbyesophagus, diabetic neuropathy and } \\
\text { Scleroderma. }\end{array}$ \\
\hline Other Causes & $\begin{array}{l}\text { latrogenic diagnosis, ingestion of lye, radiation therapy, chemotherapy, } \\
\text { intubation tracheostomy, medication related, post esophageal atresia } \\
\text { surgery or tracheoesophageal fistula surgery post-extubating }\end{array}$ \\
\hline
\end{tabular}

\subsection{Diabetes Mellitus}

In a study published in 2016, it was found that diabetes directly causes mechanophysiological changes in the esophagus. Specifically on a histological level, diabetic individuals were found to have an increased wall thickness and muscle hypertrophy, increased collagen fraction in the mucosasubmucosal layer, axonal changes in the parasympathetic fibers, and degeneration of large myelinated vagal nerve fibers. Diabetic neuropathy involves the enteric nervous system in the smooth muscle as well. These changes are demonstrated by increased esophageal wall stiffness, decreased lower esophageal sphincter tone, decreased esophageal transit, and a decrease in frequency in the peristaltic contractions after swallowing. Combined, these constellations of effects also predispose diabetic patients to developing GERD [35].

\subsection{Presbyesophagus}

Compared to younger individuals, elderly patients ( $>65$ years old) are more prone to developing dysphagia attributed to "presbyesophagus". Presbyesophagus is a term used to describe the more pronounced "physiological" changes in swallowing associated with aging [36]. The elderly population is prone to some dysfunction of both the striated and smooth muscle of the esophagus. This is explained in part by the neurological and vascular components of weakening muscle tone [37]. Figure 2 is an example of normal high resolution esophageal motility study compared to some loss of smooth muscle peristalsis in figure 3 as seen in a patient diagnosed with Presbyesophagus. 

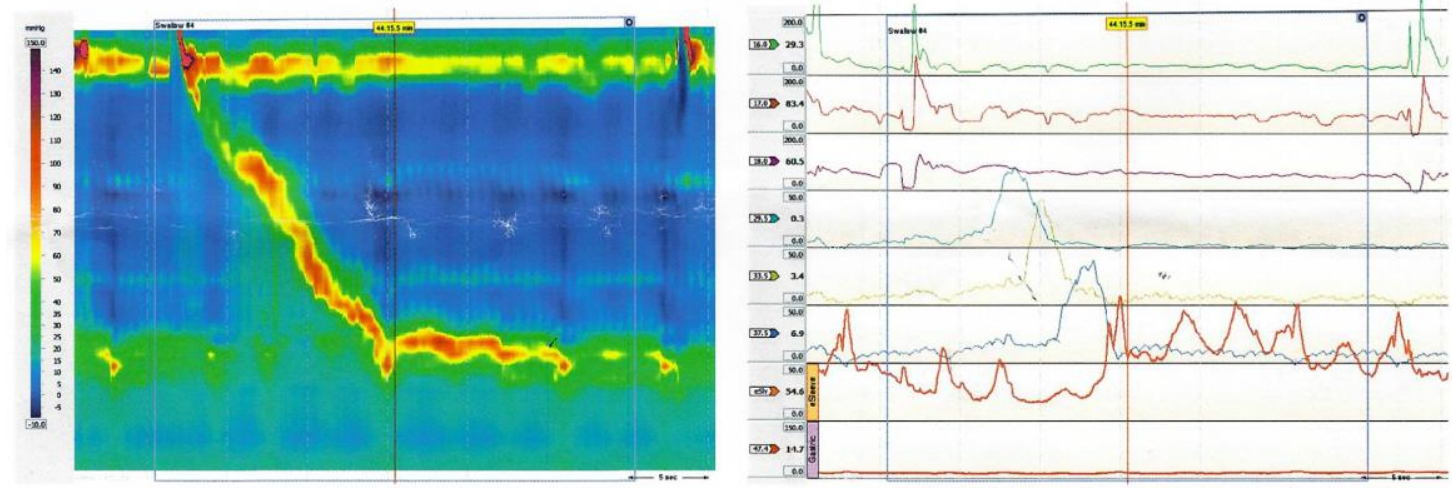

Figure 2 High-resolution esophageal motility study showing the amplitude of the peristaltic contractions are normal in the smooth muscle portion of the esophagus.
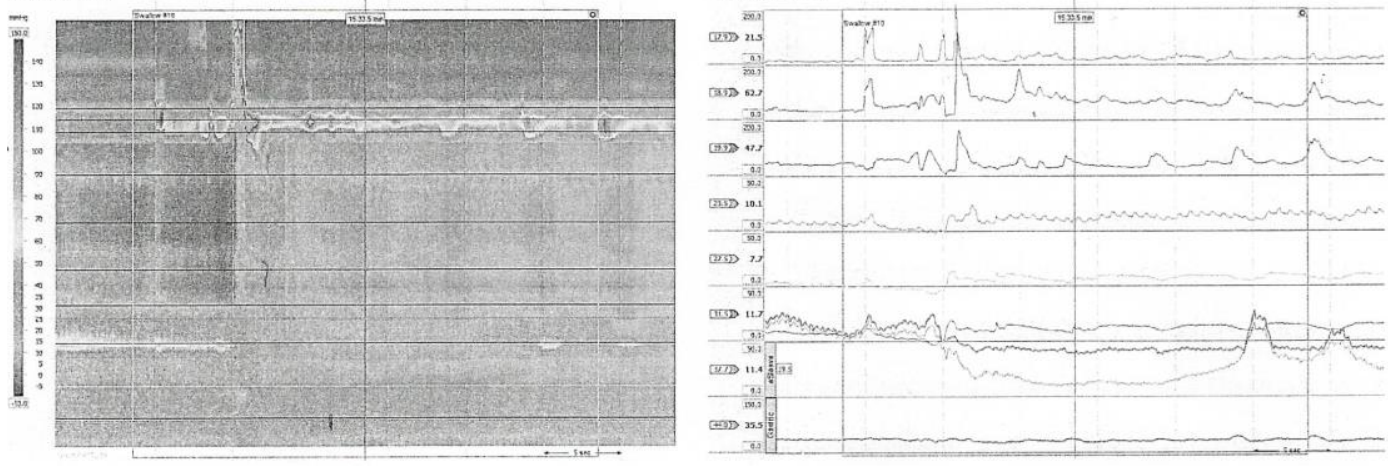

Figure 3 Presbyesophagus Manomatry: High-resolution esophageal motility study showing the smooth muscle portion of the esophagus seen here in the middle three leads has weak amplitude (less than $40 \mathrm{mmHg}$ in amplitude) and some loss of peristalsis.

\subsection{A Lower Esophageal Mucosal Ring}

A lower esophageal mucosal ring, termed "Schatzki ring" or type B ring (Figure 4) is another structural cause of dysphagia. The etiology is still of question as some have suggested it to be a congenital lesion that is always associated with a sliding hiatal hernia, while others believe that gastroesophageal reflux disease or chronic ingestion of certain medications promote the ring at the proximal margin of the sliding large hiatal hernia as the ring is not muscular, rather it is mucosal. This condition usually presents as intermittent dysphagia with solid foods, especially with bread and meat (also known as the "steak-house syndrome"). In moderate to severe cases, the dysphagia can cause significant chest pain/discomfort and sometimes the patient can present with a food impaction requiring urgent endoscopic removal for relief. Treatment for Schatzki rings is typically dilation through an endoscopic approach [38]. Figure 4 is an Endoscopic view of Schatzki ring, while Figure 5 illustrates an Endoscopic dilation with a through the scope balloon technique. 


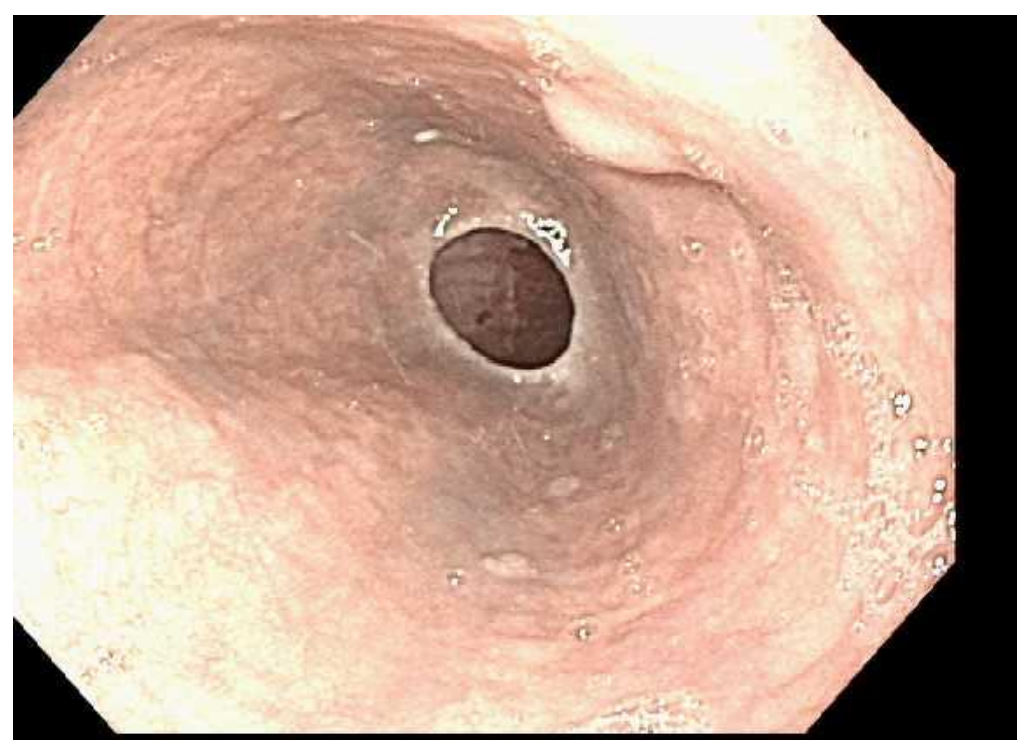

Figure 4 Endoscopic view of Schatzki ring, Type B, Located at the proximal margin of the sliding hiatal hernia which can be seen distally.

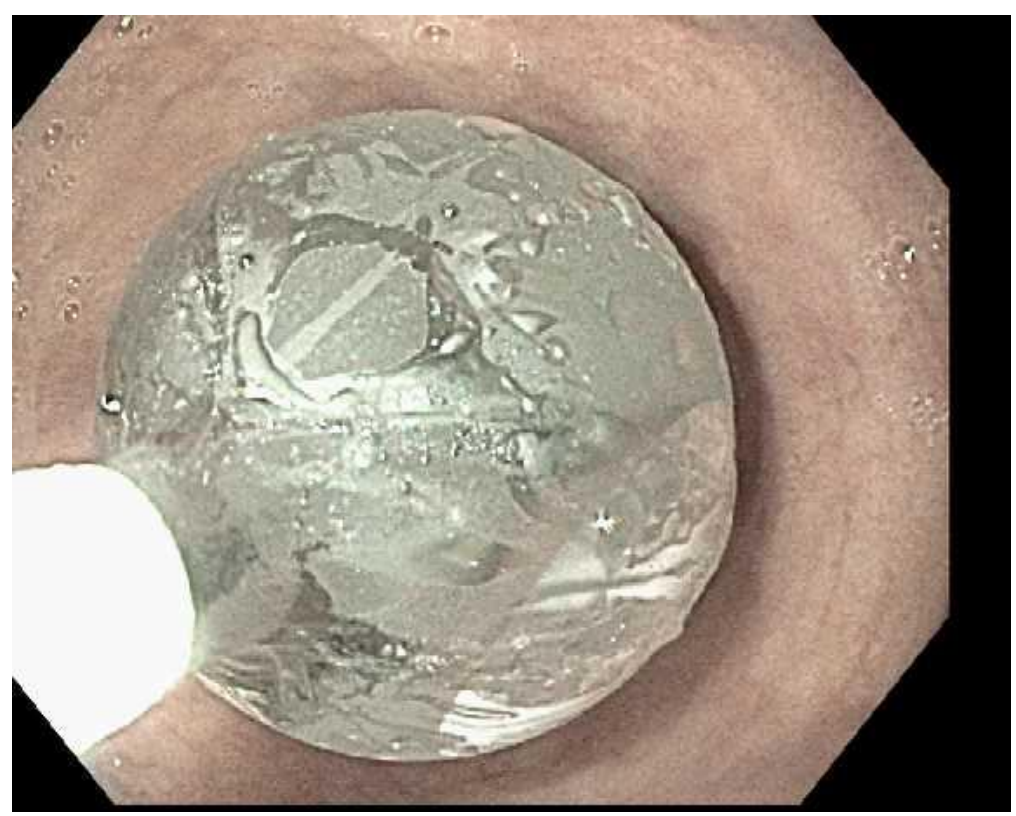

Figure 5 Endoscopic dilation of the Schatzki ring using a through the scope dilation method and a $20 \mathrm{~mm}$ diameter balloon sizing, usually requiring two separate dilation sessions of one minute each.

\subsection{Gastroesophageal Reflux Disease}

Long-standing history of gastroesophageal reflux disease (GERD), It is important to rule out esophageal strictures and even Barrett's esophagus (Figure 6). The worst scenario would be esophageal adenocarcinoma, which usually results from Barrett's esophagus. Esophageal squamous cell carcinoma more related to individuals with a significant smoking history or long-standing alcohol use, combined with weight loss or decreased appetite. If peptic ulcer strictures occur then dilation can be effective using a through the scope balloon technique or savary dilation with a guide wire, both approaches using increasing sizes of the dilation [39]. 


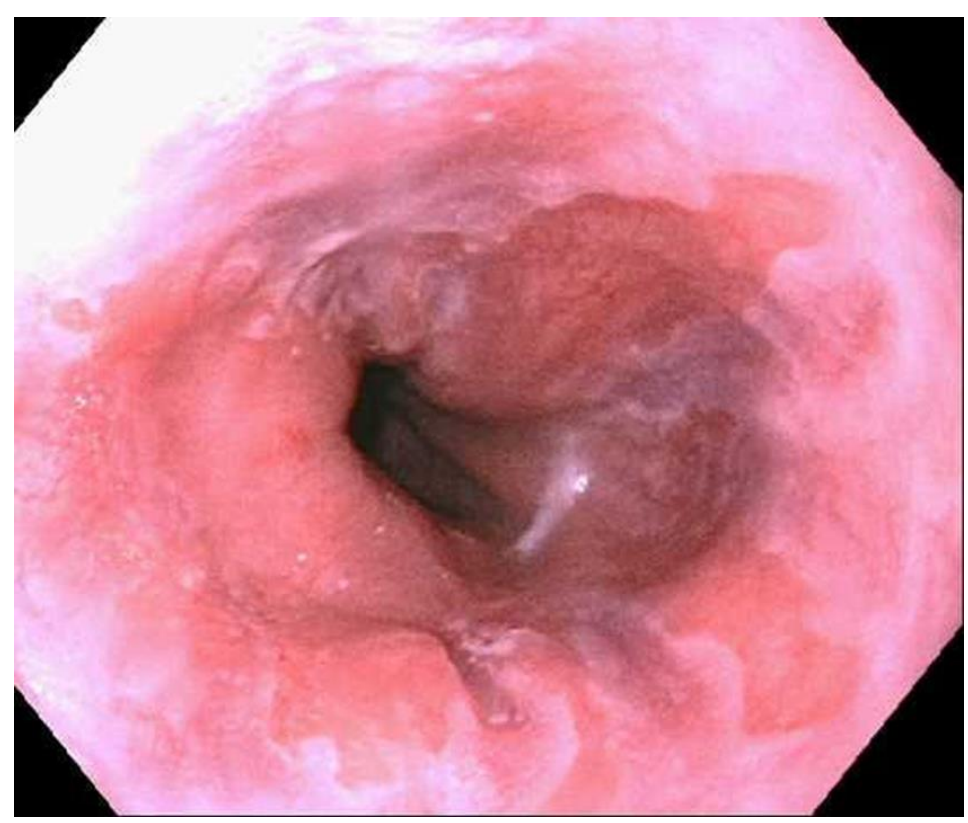

Figure 6 Endoscopic view of Barrett's esophagus. Note the irregular "tongues" of intestinal metaplasia tissue ascending into the tubular esophagus from a more circumferential area of Barrett's tissue developing from the GE reflux and the associated hiatal hernia more distally.

\subsection{Globus Pharyngeus}

Another etiology of dysphagia is a condition termed Globus Pharyngeus (formerly called Globus Hystericus). It is a persistent or intermittent feeling of a foreign object stuck in the throat, often more prominent between meals. In general patients can eat normally and multiple diagnostic tests including modified barium swallow, PH monitoring, manometry, videofluoroscopy studies, endoscopies and biopsies are normal. With limited information, studies have focused on latent GERD inducing upper esophageal sphincter spasms to protect the lungs [40]. Treatment showed improvement of the globus sensation with PPI agents [41]. Another theory that explains these symptoms is psychological factors and stress as over $96 \%$ of patients with Globus pharyngeus reported worsening of symptoms through stressful life situations [42]. Management here is with tricyclic antidepressants [43] and cognitive behavioral therapy [44].

\subsection{Extrinsic Etiologies}

Two additional but rare causes of esophageal dysphagia which result from vascular compression are dysphagia aortica and dysphagia lusoria. Dysphagia aortica is a term that is used to describe symptomatic compression of the distal esophagus by the descending thoracic aorta. This condition usually results from compression of the distal portion of the esophagus from an ectatic dilated descending aorta [45]. Dysphagia lusoria on the other hand, results from an anatomic anomaly of the right subclavian artery which posteriorly compresses the esophagus at the level of the aortic arch and is more prevalent in the elderly because of hardening of the artery by atherosclerosis [46]. 


\section{In Summary}

We have attempted to provide a comprehensive review of dysphagia in the Elderly, focusing on clinical strategies and pearls to identify the most recognized etiologies. In addition, we have addressed and summarized the less common and often "missed" etiologies. Diagnostic approaches and effective treatment options are also highlighted.

This review is also based on emphasizing the underlying physiological foundations for the processes of swallowing and esophageal transit, as well as the pathophysiologies for disease settings.

We hope this chapter is helpful at the student and resident levels as well as to our more seasoned physicians.

\section{Author Contributions}

Asaad Ishak wrote the initial draft, Data collection and editing; Richard McCallum editing the manuscript and guiding the authors; Sundar V cherukuri help with writing the manuscript, data collection and editing; Giancarlo Diaz he helped with writing the initial draft and Data collection.

\section{Competing Interests}

The authors have declared that no competing interests exist.

\section{References}

1. Sura L, Madhavan A, Carnaby G, Crary MA. Dysphagia in the elderly: Management and nutritional considerations. Clin Interv Flanging. 2012; 7: 287-298.

2. Farpour S, Farpour H, Zakeri M. Oropharyngeal dysphagia in elderly people. J Rehabil Med. 2018; 5: 120-123.

3. Park YH, Han HR, Oh BM, Lee J, Park JA, Yu SJ, et al. Prevalence and associated factors of dysphagia in nursing home residents. Geriatr Nurs. 2013; 34: 212-217.

4. Sarabia-Cobo CM, Pérez V, de Lorena P, Domínguez E, Hermosilla C, Nuñez MJ, et al. The incidence and prognostic implications of dysphagia in elderly patients institutionalized: $A$ multicenter study in Spain. Appl Nurs Res. 2016; 30: e6-e9.

5. Walton J, Silva P. Physiology of swallowing. Surgery (Oxford). 2018; 36: 529-534.

6. Aslam M, Vaezi MF. Dysphagia in the elderly. Gastroenterol Hepatol. 2013; 9: 784-795.

7. Lancaster J. Dysphagia: Its nature, assessment and management. Br J Community Nurs. 2015; 20: S28-S32.

8. Trate DM, Parkman HP, Fisher RS. Dysphagia. Evaluation, diagnosis, and treatment. Prim Care. 1996; 23: 417-432.

9. Pitchumoni CS, Dharmarajan T. Geriatric gastroenterology. New York: Springer; 2012.

10. Ekberg O, Feinberg MJ. Altered swallowing function in elderly patients without dysphagia: Radiologic findings in 56 cases. AJR Am J Roentgenol. 1991; 156: 1181-1184.

11. Forster A, Samaras N, Gold G, Samaras D. Oropharyngeal dysphagia in older adults: A review. Eur Geriatr Med. 2011; 2: 356-362.

12. Fein R, Kelsen DP, Geller N, Bains M, McCormack P, Brennan MF. Adenocarcinoma of the 
esophagus and gastroesophageal junction. Prognostic factors and results of therapy. Cancer. 1985; 56: 2512-2518.

13. Daly JM, Fry WA, Little AG, Winchester DP, McKee RF, Stewart AK, et al. Esophageal cancer: Results of an American College of Surgeons patient care evaluation study. J Am Coll Surg. 2000; 190: 562-572.

14. Novacek G. Plummer-vinson syndrome. Orphanet J Rare Dis. 2006; 1: 1-4.

15. González-Fernández M, Huckabee ML, Doeltgen SH, Inamoto Y, Kagaya H, Saitoh E. Dysphagia rehabilitation: Similarities and differences in three areas of the world. Curr Phys Med Rehabil Rep. 2013; 1: 296-306.

16. Rofes L, Arreola V, Mukherjee R, Clavé P. Sensitivity and specificity of the Eating Assessment Tool and the Volume-Viscosity Swallow Test for clinical evaluation of oropharyngeal dysphagia. Neurogastroenterol Motil. 2014; 26: 1256-1265.

17. Jaffer NM. Fluoroscopic eval of oropharyngeal dysphagia: Anatomy, technique and common etiologic factors. AJR Am J Roentgenol. 2015; 204: 49-58.

18. Gallo SH, McClave SA, Makk LJ, Looney SW. Standardization of clinical criteria required for use of the 12.5 millimeter barium tablet in evaluating esophageal lumenal patency. Gastrointest Endosc. 1996; 44: 181-184.

19. Langmore SE. Endoscopic evaluation of oral and pharyngeal phases of swallowing. GI Motil Online. 2006. doi:10.1038/gimo28.

20. Sanyaolu LN, Jemah A, Stew B, Ingrams DR. The role of transnasal oesophagoscopy in the management of globus pharyngeus and non-progressive dysphagia. Ann R Coll Surg Engl. 2016; 98: 49-52.

21. Sood A, Gambhir S, Singh D, Kumar KU, Parihar AS, Kheruka S, et al. In-house-prepared semisolid bolus for esophageal transit scintigraphy in normal volunteers and its comparison with liquid bolus. Indian J Nucl Med. 2020; 35: 130-135.

22. Alfonsi E, Restivo DA, Cosentino G, De Icco R, Bertino G, Schindler A, et al. Botulinum toxin is effective in the management of neurogenic dysphagia. Clinical-electrophysiological findings and tips on safety in different neurological disorders. Front Pharmacol. 2017; 8: 80.

23. Alfonsi E, Merlo IM, Ponzio M, Montomoli C, Tassorelli C, Biancardi C, et al. An electrophysiological approach to the diagnosis of neurogenic dysphagia: Implications for botulinum toxin treatment. J Neurol Neurosurg Psychiatry. 2010; 81: 54-60.

24. Barer $\mathrm{DH}$. The natural history and functional consequences of dysphagia after hemispheric stroke. J Neurol Neurosurg Psychiatry. 1989; 52: 236-241.

25. Martino R, Foley N, Bhogal S, Diamant N, Speechley M, Teasell R. Dysphagia after stroke: Incidence, diagnosis, and pulmonary complications. Stroke. 2005; 36: 2756-2763.

26. Dennis M. Nutrition after stroke. Br Med Bull. 2000; 56: 466-475.

27. Kwon M, Lee JH. Oro-pharyngeal dysphagia in Parkinson's disease and related movement disorders. J Mov Disord. 2019; 12: 152-160.

28. Mu L, Sobotka S, Chen J, Su H, Sanders I, Adler CH, et al. Altered pharyngeal muscles in Parkinson disease. J Neuropathol Exp Neurol. 2012; 71: 520-530.

29. Valdez IH, Atkinson JC, Ship JA, Fox PC. Major salivary gland function in patients with radiationinduced xerostomia: Flow rates and sialochemistry. Int J Radiat Oncol Biol Phys. 1993; 25: 4147.

30. Vivino FB. Sjogren's syndrome: Clinical aspects. Clin Immunol. 2017; 182: 48-54. 
31. Wiseman LR, Faulds D. Oral pilocarpine: A review of its pharmacological properties and clinical potential in xerostomia. Drugs. 1995; 49: 143-155.

32. Chandrakala B, Sumathy G. Killian Jamieson diverticulum. Eur J Mol Clin Med. 2020; 7: 13271330.

33. Ferreira LEVVC, Simmons DT, Baron TH. Zenker's diverticula: Pathophysiology, clinical presentation, and flexible endoscopic management. Dis Esophagus. 2008; 21: 1-8.

34. Wang AY, Kadkade R, Kahrilas PJ, Hirano I. Effectiveness of esophageal dilation for symptomatic cricopharyngeal bar. Gastrointest Endosc. 2005; 61: 148-152.

35. Zhao J, Gregersen H. Diabetes-induced mechanophysiological changes in the esophagus. Ann N Y Acad Sci. 2016; 1380: 139-154.

36. Dumic I, Nordin T, Jecmenica M, Stojkovic Lalosevic M, Milosavljevic T, Milovanovic T. Gastrointestinal tract disorders in older age. Can J Gastroenterol Hepatol. 2019; 2019. doi:10.1155/2019/6757524

37. Logrippo S, Ricci G, Sestili M, Cespi M, Ferrara L, Palmieri GF, et al. Oral drug therapy in elderly with dysphagia: Between a rock and a hard place! Clin Interv Aging. 2017; 12: 241-251.

38. Jalil S, Castell DO. Schatzki's ring: A benign cause of dysphagia in adults. J Clin Gastroenterol. 2002; 35: 295-298.

39. Egan JV, Baron TH, Adler DG, Davila R, Faigel DO, Gan SL, et al. Esophageal dilation. Gastrointest Endosc. 2006; 63: 755-760.

40. Lee BE, Kim GH. Globus pharyngeus: A review of its etiology, diagnosis and treatment. World J Gastroenterol. 2012; 18: 2462.

41. Divi V, Benninger MS. Diagnosis and management of laryngopharyngeal reflux disease. Curr Opin Otolaryngol Head Neck Surg. 2006; 14: 124-127.

42. Harris MB, Deary IJ, Wilson JA. Life events and difficulties in relation to the onset of globus pharyngis. J Psychosom Res. 1996; 40: 603-615.

43. Cybulska EM. Globus hystericus-a somatic symptom of depression? The role of electroconvulsive therapy and antidepressants. Psychosom Med. 1997; 59: 67-69.

44. Cathcart R, Wilson JA. Lump in the throat. Clin Otolaryngol. 2007; 32: 108-110.

45. Mittal RK, Siskind BN, Hongo M, Flye MW, McCallum RW. Dysphagia aortica. Dig Dis Sci. 1986; 31: 379-384.

46. Berenzweig $H$, Baue AE, McCallum RW. Dysphagia lusoria. Report of a case and review of the diagnostic and surgical approach. Dig Dis Sci. 1980; 25: 630-636. 


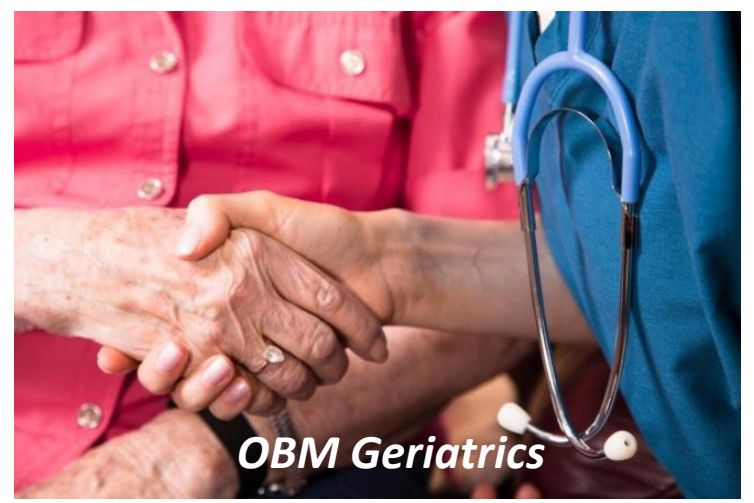

Enjoy $O B M$ Geriatrics by:

1. Submitting a manuscript

2. Joining in volunteer reviewer bank

3. Joining Editorial Board

4. Guest editing a special issue

For more details, please visit: http://www.lidsen.com/journals/geriatrics 